\title{
sciforum
}

Conference Proceedings Paper

\section{Silicon Self-Diffusion in Stishovite: Calculations of Point Defect Parameters Based on the $\mathrm{cB} \Omega$ Thermodynamic Model}

\author{
V. Saltas ${ }^{1, *}$ and F. Vallianatos ${ }^{1,2}$ \\ 1 Institute of Physics of Earth's Interior and Geohazards, and UNESCO Chair in Solid Earth Physics and \\ Geohazards Risk Reduction, Hellenic Mediterranean University Research Center, Crete, Greece; \\ saltas@hmu.gr (V.S.) \\ 2 Department of Geology and Geoenvironment, National and Kapodistrian University of Athens, Greece; \\ fvallian@geol.uoa.gr (F.V.) \\ * Correspondence: saltas@hmu.gr (V.S.)
}

Received: date; Accepted: date; Published: date

\begin{abstract}
In the present work we apply the $c B \Omega$ thermodynamic model to study the diffusion of $\mathrm{Si}$ in stishovite crystal at high pressure and in a wide temperature range. According to this model, the point defect activation Gibbs free energy is expressed as a function of the bulk properties of the material, i.e. $\mathrm{g}^{\text {act }}=c B \Omega$, where $B$ is the isothermal bulk modulus, $\Omega$ is the mean atomic volume and $\mathrm{c}$ is a dimensionless constant. In this way, other important point defect parameters, such as the activation volume $v^{\text {act }}$, the activation entropy $s^{\text {act }}$ and the activation enthalpy $h^{\text {act }}$ may be estimated, if the thermoelastic properties of the material are known over a wide temperature and pressure range. Our calculations are based on previously reported self-diffusion coefficients in stishovite single crystals measured at $14 \mathrm{GPa}$ and at temperatures from 1400 to $1800{ }^{\circ} \mathrm{C}$, in the [110] and [001] directions, by Shatskiy et al. [Am. Mineral. 2010, 95, 135-43]. Furthermore, the EOS of stishovite, proposed by Wang et al. [J. Geophys. Res. 2012, 117, B06209] has been used for the accurate implementation of the $c B \Omega$ model. Our results suggest that the aforementioned point defect parameters exhibit considerable temperature dependence over the studied temperature range $\left(1000-2000{ }^{\circ} \mathrm{C}\right)$. The estimated activation volumes $\left(4.4-5.3 \mathrm{~cm}^{3} / \mathrm{mol}\right.$, in the range $\left.1400-1800{ }^{\circ} \mathrm{C}\right)$ are in agreement with reported experimental results. Our study confirms the potential of the $c B \Omega$ model for the theoretical investigation of diffusion processes in minerals, in order to overcome the experimental difficulties and the lack of experimental diffusion data in mantle conditions.
\end{abstract}

Keywords: stishovite; self-diffusion; thermodynamic modelling; activation volume; high temperature; high pressure

\section{Introduction}

Stishovite is a high-pressure polymorph of $\mathrm{SiO}_{2}$, with the tetragonal rutile structure $\left(\mathrm{P}_{2} / \mathrm{mnm}\right.$, space group 136). Stishovite is hardly found on Earth's surface but is the predominant form of silica in the lower mantle [1]. The key role of stishovite as a potential mineral that stores water in its crystal structure and transports it into the deep mantle has been recently clarified [2]. Furthermore, the appearance of stishovite in $\mathrm{SiO}_{2}$-rich fragments has been proposed to explain the presence of seismic reflectors in the mid-mantle region [3]. These seismic reflectors would be affected by the rheological properties of the related materials undergoing plastic deformation under mantle conditions. Plastic deformation occurs either by diffusion or dislocation creep which are both controlled by atomic diffusion [3,4]. Silicon self-diffusion in stishovite was studied for the first time by Shatskiy et al. at 
the temperature range, $1400-1800{ }^{\circ} \mathrm{C}$ and at $14 \mathrm{GPa}$, after their successful synthesis of large single crystals [5,6]. Their results suggest a weak anisotropy of diffusion along the [001] and [110] directions. Based on these experimental diffusion data, in the present work, we apply the so-called $\mathrm{cB} \Omega$ thermodynamic model [7] to further estimate important point defect parameters of Si self-diffusion in stishovite. According to this model, proposed by Varotsos and Alexopoulos [8,9], the diffusion coefficients of point defects can be calculated if the thermoelastic properties of the host material are known. A concise description of the model is given in the next section.

The $c B \Omega$ thermodynamic model has been applied successfully over the past years to study selfor hetero-diffusion in a wide range of materials, such as metals, noble gas solids, alkali halides, diamond, oxides, semiconductors ( $\mathrm{Si}, \mathrm{Ge}, \mathrm{GaAs}, \mathrm{Si}_{1-\mathrm{x}} \mathrm{Ge}_{\mathrm{x}}$ ) and minerals [10-20]. However, diffusion in minerals has been less studied in the framework of the $c B \Omega$ model, as compared to other materials, probably due to the lack of complete sets of thermoelastic properties necessary to implement the model at temperatures and pressures, similar to that of the Earth's interior. Systematic studies of diffusion in minerals in the framework of the $c B \Omega$ model have been carried out by Zhang et al. $[12,14]$ who have studied oxygen self-diffusion in silicate and oxide minerals, and $H$, $\mathrm{Na}$ and $\mathrm{K}$ diffusion in plagioclase feldspar. Notably, the model has been also used to explain the emission of seismic electrical signals (SES) as precursors of earthquake events and to describe the thermodynamical and rheological properties of Earth's mantle [21,22].

In the present case, we apply the capabilities of the model in conjunction with recent EOS of stishovite to calculate important point defect parameters of Si self-diffusion, such as the activation enthalpy, activation entropy and activation volume at 14 GPa, in the temperature range, 1000-2000 ${ }^{\circ} \mathrm{C}$.

\section{Methodology}

\subsection{The $c B \Omega$ thermodynamic model}

The basis of the $c B \Omega$ model lies in the substantiated assumption that the Gibbs free energy of an activation process such as the formation and migration of point defects is expressed as a function of the bulk properties of the host material, according to the relation:

$$
g^{a c t}=c^{a c t} B \Omega
$$

where $B$ is the isothermal bulk modulus, $\Omega$ is the mean volume per atom and $c^{\text {act }}$ is a dimensionless constant, independent of temperature and pressure, under certain conditions [7]. As a consequence, in the case of a single self- or hetero-diffusion process, the diffusion coefficients $D$ are expressed via the well-known Arrhenius equation, as follows:

$$
D=D_{o} \exp \left(-c^{a c t} B \Omega / k_{\mathrm{B}} T\right)
$$

where the pre-exponential factor, $D_{o}$ depends on the structure and the diffusion mechanism, the jump distance and the corresponding jump frequency of the defect species [7-9].

Based on Equation (1), the temperature dependence of important point defect parameters such as the activation entropy $s^{a c t}$, activation enthalpy $h^{\text {act }}$ and activation volume $v^{\text {act }}$, are determined from the following equations:

$$
\begin{gathered}
\left.\left.s^{a c t}=-\frac{\partial g^{a c t}}{\partial T}\right]_{P}=-c^{a c t} \Omega\left\{\frac{\partial B}{\partial T}\right]_{P}+\beta B\right\} \\
\left.h^{a c t}=g^{a c t}+T \cdot s^{a c t}=c^{a c t} \Omega\left\{B-T \beta B-T \frac{\partial B}{\partial T}\right]_{P}\right\}
\end{gathered}
$$

and

$$
\left.\left.v^{a c t}=\frac{\partial g^{a c t}}{\partial P}\right]_{T}=c^{a c t} \Omega\left\{\frac{\partial B}{\partial P}\right]_{T}-1\right\}
$$


where $\beta$ is the thermal volume expansion coefficient. If the thermoelastic properties of the material are known, the temperature (and pressure) dependence of these important parameters are derived.

In order to calculate the abovementioned quantities, the constant $c^{\text {act }}$ should be firstly estimated. An accurate method is to calculate the mean value of $c^{\text {act }}$ under the condition that sufficient experimental diffusion data are available over a broad temperature range [13]. Taking the natural logarithm of both sides in Equation (2), we get:

$$
\ln D=\ln D_{o}-c^{a c t} \frac{B \Omega}{k_{B} T}
$$

The linear dependence of experimental $\ln D$ versus $B \Omega / k_{B} T$ suggests the validity of the $c B \Omega$ model with respect to a single diffusion mechanism; while, it leads directly to the estimation of $c^{\text {act }}$ from the slope of the linear fit [18].

\subsection{Bulk properties of stishovite}

For the implementation of the $c B \Omega$ model to the diffusion of $\mathrm{Si}$ in stishovite, according to Equations (1)-(5), the thermoelastic properties of stishovite should be known over the desired temperature range. The $P-V-T$ EOS of stishovite in the temperature range, $300-1700 \mathrm{~K}$ and at pressures from 17 to $54 \mathrm{GPa}$, i.e., under lower mantle conditions, has been determined by Wang et al., who carried out measurements in a Kawai-type multi-anvil apparatus [23]. In their study, Wang et al. used the Mie-Grüneisen-Debye (MGD) model to fit their experimental data and the third order Birch-Murnaghan and Vinet equations for the $300 \mathrm{~K}$ isotherms. Important thermoelastic properties such as density, Grüneisen parameter, isothermal and adiabatic bulk modulus, thermal expansion coefficient and isobaric heat capacity were calculated from ambient pressure up to $70 \mathrm{GPa}$ and in the temperature range, $300-2500 \mathrm{~K}$.

Based on these latest reported results, we used second order polynomial fittings to interpolate the values of $B(T), \Omega(T)$ and $\beta(T)$ at the pressure of $14 \mathrm{GPa}$, where the diffusion measurements by Shatskiy et al. were carried out [5,6], and in the temperature range, $1000-2000{ }^{\circ} \mathrm{C}$ that we used in the present study. Our calculations of $B, \Omega$ and $\beta$ at $14 \mathrm{GPa}$ in the temperature range $1000-2000{ }^{\circ} \mathrm{C}$ are shown in Figure 1. Furthermore, the temperature and pressure derivatives of the isothermal bulk modulus $\left.\left.B(\partial B / \partial T]_{P}, \partial B / \partial P\right]_{T}\right)$ were also calculated at $14 \mathrm{GPa}$. The temperature derivative of $B$, $\partial B / \partial T]_{P}$ varies linearly from $-0.059 \mathrm{GPa} / \mathrm{K}$ to $-0.074 \mathrm{GPa} / \mathrm{K}$, in the range $1000-2000{ }^{\circ} \mathrm{C}$. The pressure derivative of $B$ follows a quadratic behavior with temperature, according to the relation, $\partial B / \partial P]_{T}=$ $4.63+8.20 \times 10^{-5} T+2.52 \times 10^{-7} T^{2}$. Thus, in the studied range from 1000 to $2000{ }^{\circ} \mathrm{C}$, the pressure derivative of $B$ increases from 5.14 to 6.11 , respectively.

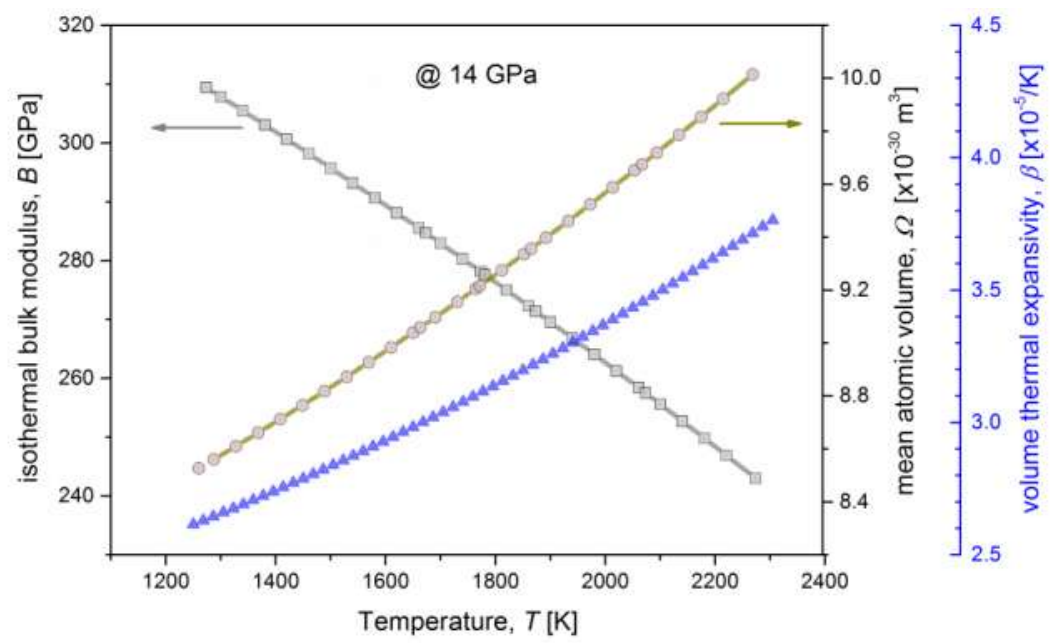

Figure 1. Isothermal bulk modulus $B$, mean atomic volume $\Omega$ and volume thermal expansivity $\beta$, at $14 \mathrm{GPa}$, in the temperature range, $1000-2000^{\circ} \mathrm{C}$, calculated on the basis of the $P-V-T$ EOS of stishovite reported by Wang et al. [23]. 


\section{Results and Discussion}

The diffusion of $\mathrm{Si}$ in the [110] and [001] crystallographic directions of stishovite has been studied by Shatskiy et al. at $14 \mathrm{GPa}$, in the temperature range, $1400-1800{ }^{\circ} \mathrm{C}[5,6]$. They reported that the temperature dependence of the diffusion coefficients for each direction is expressed through the following Arrhenius relations:

$$
\begin{aligned}
& D_{\left[\begin{array}{ll}
1 & 1
\end{array}\right] 0}=4.10 \times 10^{-1} \exp \left(\frac{-322 \pm 28(\mathrm{~kJ} / \mathrm{mol})}{R T}\right) \mathrm{m}^{2} / \mathrm{s}, \\
& \left.D_{[0} 0\right] 11=5.62 \times 10^{-1} \exp \left(\frac{-334 \pm 39(\mathrm{~kJ} / \mathrm{mol})}{R T}\right) \mathrm{m}^{2} / \mathrm{s}
\end{aligned}
$$

The reported experimental values of the Si self-diffusion coefficients $D$ in logarithmic scale as a function of the dimensionless quantity $B \Omega / k_{B} T$ are shown in Figure 2 . The linear dependence of $\log D$ versus $B \Omega / k_{\mathrm{B}} T$ implies the validity of the $c B \Omega$ model, according to Equation (6). From the linear fittings of the data, the parameter $c^{\text {act }}$ has been extracted for the two crystallographic directions (refer to Table 1). Obviously, these values are very close to each other, due to the similar slopes of the two lines, as shown in Figure 2. Similar values of $c^{\text {act }}$ have been reported for He diffusion in forsterite and olivine, i.e. $0.160-0.189$, depending on the crystallographic direction of diffusion [13].

Proceeding further, we calculated the temperature dependence of point defect thermodynamic properties, $g^{a c t}, s^{a c t}, h^{a c t}$ and $v^{a c t}$ at $14 \mathrm{GPa}$, where the diffusion experiments of Si-diffusion in stishovite were carried out [5,6]. Given that the reported diffusion experiments were conducted over the range, $1400-1800{ }^{\circ} \mathrm{C}$, we extended our calculations to the temperature range, $1000-2000{ }^{\circ} \mathrm{C}$. The calculated values, according to the Equations (1) and (3)-(5), are shown in Figure 3. We observe that all the aforementioned quantities exhibit considerable change with temperature. The ranges of the calculated values with their uncertainties are summarized in Table 1.

Table 1. Calculated values of $c^{a c t}$, activation enthalpy, activation entropy, activation Gibbs free energy and activation volume for Si diffusion in stishovite. Reported experimental values of activation enthalpy and activation volume are also included.

\begin{tabular}{cccccccc}
\hline direction & $c^{\text {act }}$ & $\begin{array}{c}h_{\text {calc }}^{\text {act }} \\
(\mathrm{kJ} / \mathrm{mol})\end{array}$ & $\begin{array}{c}h_{\text {exp }}^{\text {act }} \\
(\mathrm{kJ} / \mathrm{mol})\end{array}$ & $\begin{array}{c}s^{\text {act }} \\
(k \mathrm{~B} \text { units })\end{array}$ & $\begin{array}{c}g^{\text {act }} \\
(\mathrm{kJ} / \mathrm{mol})\end{array}$ & $\begin{array}{c}v_{\text {calc }}^{\text {act }} \\
\left(\mathrm{cm}^{3} / \mathrm{mol}^{2}\right)\end{array}$ & $\begin{array}{c}v_{\text {exp }}^{\text {act }} \\
\left(\mathrm{cm}^{3} / \mathrm{mol}^{2}\right)\end{array}$ \\
\hline$[110]$ & $0.182 \pm 0.016$ & $(350-429) \pm 19$ & $322 \pm 28^{1}$ & $(5.7-8.6) \pm 0.3$ & $(289-267) \pm 17$ & $(3.9-5.6) \pm 0.6$ & $6.0 \pm 1.0^{2}$ \\
{$[001]$} & $0.188 \pm 0.021$ & $(359-438) \pm 21$ & $334 \pm 39^{1}$ & $(5.9-8.9) \pm 0.3$ & $(299-276) \pm 17$ & $(4.0-5.8) \pm 0.6$ & - \\
\hline
\end{tabular}

${ }^{1}$ Ref. [5], ${ }^{2}$ Ref. [3].

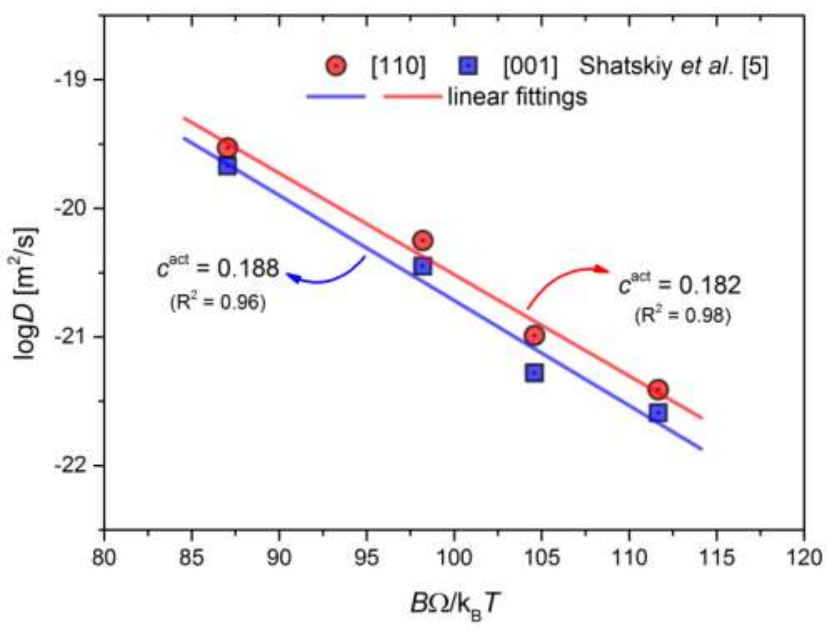

Figure 2. Experimental diffusion coefficients of $\mathrm{Si}$ in stishovite [5,6] for the two crystallographic directions, [110] and [001], as a function of the dimensionless quantity $B \Omega / k_{\mathrm{B}} T$. From the linear fit of the lines, the parameter $c^{a c t}$ is derived in each case. The correlation factors, $\mathrm{R}^{2}$ are also given in parentheses. 


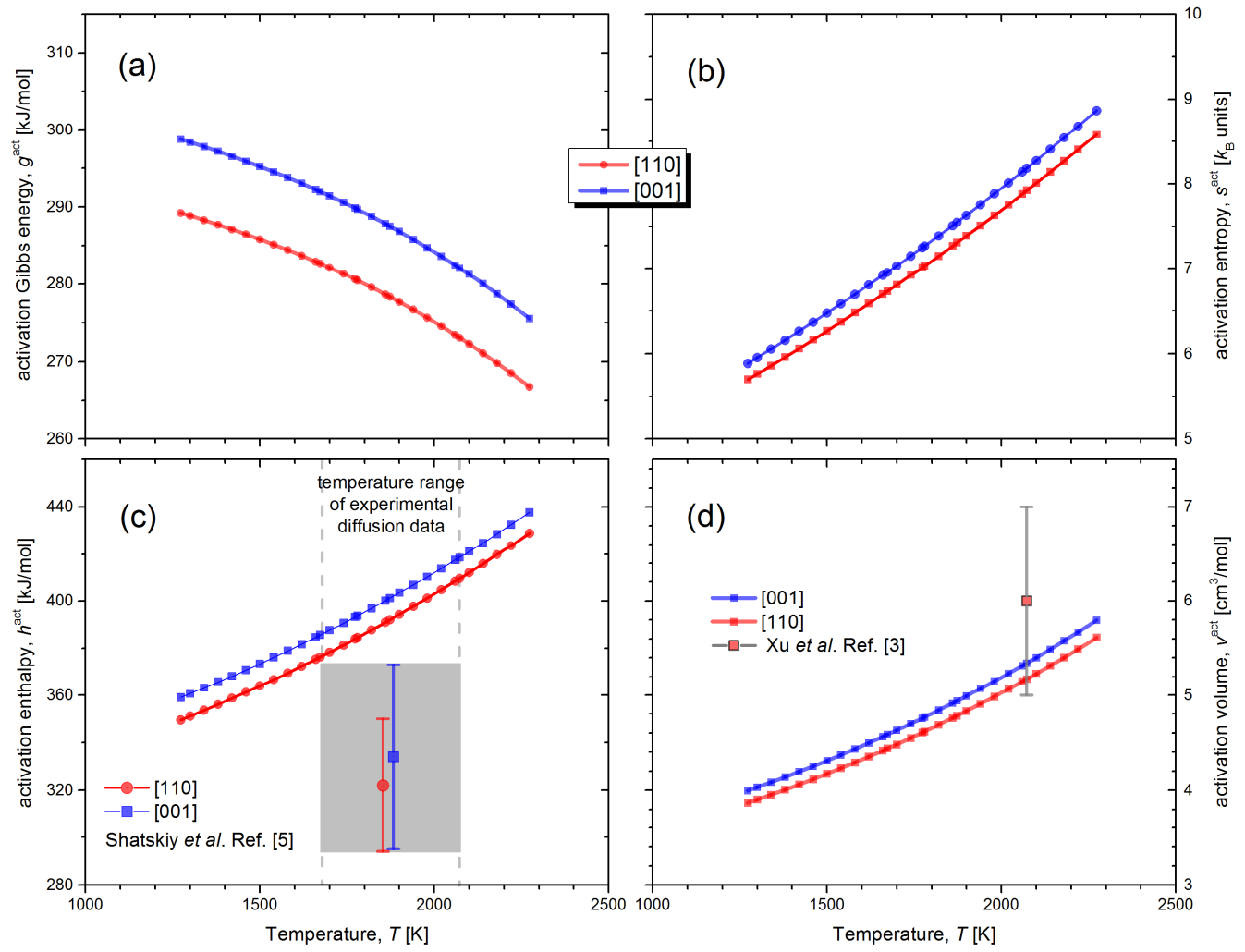

Figure 3. Calculated point defect parameters of Si self-diffusion across the [110] and [001] crystallographic directions in stishovite (a) Activation Gibbs free energy, $g^{\text {act }}$; (b) Activation entropy, $s^{a c t} ;$ (c) Activation enthalpy, $h^{a c t}$ and (d) Activation volume, $v^{\text {act }}$. Reported experimental values of activation enthalpy and activation volume have been also included in the plots.

The observed significant temperature variation of the calculated point defect parameters (refer to Figure 3 and Table 1) is attributed to the non-linear behavior of the isothermal bulk modulus and the mean atomic volume of stishovite, as shown in Figure 1, according to the corresponding EOS reported by Wang et al. [23]. Indeed, when linear approximations have been considered to describe the temperature dependence of the bulk modulus and the mean atomic volume over a narrow temperature range or at lower temperatures compared to the present case, no considerable variation of activation enthalpy and activation volume has been observed in the application of the $c B \Omega$ model. These are the cases of He diffusion in olivine and forsterite [13], and of tin diffusion in Ge [24], where $h^{\text {act }}$ and $v^{\text {act }}$ actually remain constant with temperature. On the contrary, in the case of self- and hetero-diffusion in $\mathrm{Si}$, the anharmonic behavior of the bulk modulus of silicon has resulted in considerable variation of the activation enthalpy (3.6-4.9 eV for Si self-diffusion) [15,18,19]. The idea of linearly varying activation enthalpy with temperature was arbitrarily used by Kube et al. [25] to explain their experimental findings of the non-linear Arrhenius behavior of self-diffusion coefficients in Si. However, the above assumption has been explained on the basis of the $c B \Omega$ model by considering the anharmonicity of the bulk properties of Si $[15,19]$.

In the present case, our calculations of $h^{\text {act }}$ are in the range $350-429 \mathrm{~kJ} / \mathrm{mol}$ (refer to Table 1) which are slightly overestimated as compared to the experimental values, i.e. $(322 \pm 28) \mathrm{kJ} / \mathrm{mol}$ and $(334 \pm 39) \mathrm{kJ} / \mathrm{mol}$, for the [110] and [001] directions, respectively [5,6]. The calculated values of $v^{\text {act }}$ $\left(4.4-5.3 \mathrm{~cm}^{3} / \mathrm{mol}\right)$ in the range $1400-1800{ }^{\circ} \mathrm{C}$ are comparable to the reported value of $(6.0 \pm 1.0) \mathrm{cm}^{3} / \mathrm{mol}$, taking into account the experimental uncertainties. The above calculated values of $v^{\text {act }}$ are close to the mean atomic volume $\Omega_{\mathrm{o}}$, calculated at $1000{ }^{\circ} \mathrm{C}\left(8.53 \times 10^{-30} \mathrm{~m}^{3}\right)$. Specifically, $v^{\text {act }}$ varies from 0.89 to $1.04 \Omega_{\mathrm{o}}$, in the range $1400-1800{ }^{\circ} \mathrm{C}$, where the diffusion experiments in stishovite were carried out by Shatskiy et al. [5]. It is worth mentioning that the sign and value of activation volume is indicative 
of the involved type of diffusion mechanism, namely vacant or interstitial sites. Values of activation volume comparable to the mean atomic volume probably suggests vacancy-mediated self-diffusion in stishovite $[15,19]$.

\section{Conclusions}

In the present study, Si self-diffusion in stishovite was studied at high pressure (14 GPa) in the temperature range $1000-2000{ }^{\circ} \mathrm{C}$, on the basis of the $c B \Omega$ thermodynamic model which connects the thermodynamic properties of point defects with the thermoelastic properties of the host material. The calculated activation parameters of diffusion, namely, activation Gibbs free energy, activation entropy, activation enthalpy and activation volume follow a monotonic temperature dependence, which can be attributed to the anharmonic behavior of the bulk properties of stishovite. Our calculations of activation enthalpy and activation volume are in good agreement with reported experimental diffusion data of self-diffusion in stishovite. The calculated activation volume is close to the mean atomic volume which is compatible with vacancy-mediated self-diffusion in stishovite.

Our study confirms the potential of the $c B \Omega$ model for the theoretical investigation of self- and hetero-diffusion in minerals, in order to overcome the experimental difficulties and the lack of experimental diffusion data under high- $P$ and high- $T$ conditions.

Author Contributions: Conceptualization, V.S.; methodology, V.S. and F.V.; validation, V.S. and F.V.; formal analysis, V.S.; resources, V.S.; writing-original draft preparation, V.S.; writing-review and editing, V.S. and F.V. All authors have read and agreed to the published version of the manuscript.

Funding: We acknowledge financial support of this work by the project "HELPOS-Hellenic System for Lithosphere Monitoring, Greece" (MIS 5002697) which is implemented under the Action "Reinforcement of the Research and Innovation Infrastructure", funded by the Operational Programme "Competitiveness, Entrepreneurship and Innovation, Greece" (NSRF2014-2020) and co-financed by Greece and the European Union (European Regional Development Fund).

Conflicts of Interest: The authors declare no conflict of interest.

\section{References}

1. Nishihara, Y., Nakayama, K., Takahashi, E., Iguchi, T., Funakoshi, K. ìchi. P-V-T equation of state of stishovite to the mantle transition zone conditions, Phys. Chem. Miner., 2005, 31(10), 660-70, Doi: 10.1007/s00269-004-0426-7.

2. Lin, Y., Hu, Q., Meng, Y., Walter, M., Mao, H.K. Evidence for the stability of ultrahydrous stishovite in Earth's lower mantle, Proc. Natl. Acad. Sci., 2020, 117(1), 184-9, Doi: 10.1073/pnas.1914295117.

3. Xu, F., Yamazaki, D., Sakamoto, N., Sun, W., Fei, H., Yurimoto, H. Silicon and oxygen self-diffusion in stishovite: Implications for stability of $\mathrm{SiO}_{2}$-rich seismic reflectors in the mid-mantle, Earth Planet. Sci. Lett. 2017, 459, 332-9, Doi: 10.1016/j.epsl.2016.11.044.

4. Karato, S.-I. Rheological Properties of Minerals and Rocks. In Physics and Chemistry of the Deep Earth, 2013, S.-I. Karato (Ed.). doi:10.1002/9781118529492.ch4.

5. Shatskiy, A., Yamazaki, D., Borzdov, Y.M., Matsuzaki, T., Litasov, K.D., Cooray, T., Ferot, A., Ito, E., Katsura, T. Stishovite single-crystal growth and application to silicon self-diffusion measurements, Am. Mineral. 2010, 95, 135-43, Doi: 10.2138/am.2010.3255.

6. Katsura's Lab/High-Pressure Research in Earth Science. Available online: https://katsurabgi.jimdo.com/ english-home/lecture-note/past-studies/silicon-self-diffusion-in-stishovite/ (accessed on 5 Sept. 2020).

7. Varotsos, P. and Alexopoulos K. Thermodynamics of Point Defects and their Relation with the Bulk Properties, North-Holland, Amsterdam, 1986.

8. Varotsos, P. and Alexopoulos, K. Calculation of diffusion coefficients at any temperature and pressure from a single measurement. I. Self diffusion. Phys. Rev. B 1980, 22, 3130-4, Doi:10.1103/PhysRevB.22.3130.

9. Varotsos, P. and Alexopoulos, K. Calculation of diffusion coefficients at any temperature and pressure from a single measurement. II. Heterodiffusion. Phys. Rev. B 1981, 24, 3606-9, doi:10.1103/PhysRevB.24.3606.

10. Varotsos, P. and Alexopoulos, K. Connection between the formation volume and formation Gibbs energy in noble-gas solids. Phys. Rev. B, 1984, 30, 7305-7306, Doi: 10.1103/PhysRevB.30.7305 
11. Varotsos, P. Comparison of models that interconnect point defect parameters in solids with bulk properties. J. Appl. Phys. 2007, 101, 123503-8, Doi: 10.1063/1.2745359.

12. Zhang, B., $\mathrm{Wu}, \mathrm{X} ., \mathrm{Xu}, \mathrm{J}$., Zhou, R. Application of the $\mathrm{cB} \Omega$ model for the calculation of oxygen self-diffusion coefficients in minerals, J. Appl. Phys., 2010, 108(5), 053505-6, Doi: 10.1063/1.3476283.

13. Vallianatos, F., Saltas, V. Application of the $\mathrm{cB} \Omega$ model to the calculation of diffusion parameters of He in olivine, Phys. Chem. Miner. 2014, 41, 181-188, Doi: 10.1007/s00269-013-0636-y.

14. Zhang, B., Shan, S., Wu, X. Modeling H, Na, and $\mathrm{K}$ diffusion in plagioclase feldspar by relating point defect parameters to bulk properties, Phys. Chem. Miner., 2016, 43(2), 151-9, Doi: 10.1007/s00269-015-0782-5.

15. Saltas, V., Chroneos, A., Vallianatos, F. A thermodynamic approach to self-diffusion in silicon: Evidence of a single diffusion mechanism?, Mater. Chem. Phys., 2016, 181, 204-208, Doi: 10.1016/ j.matchemphys.2016.06.050.

16. Saltas, V., Chroneos, A., Cooper, M.W.D., Fitzpatrick, M.E., Vallianatos, F. Investigation of oxygen self-diffusion in $\mathrm{PuO}_{2}$ by combining molecular dynamics with thermodynamic calculations, RSC Adv., 2016, 6(105), 103641-9, Doi: 10.1039/c6ra24575g.

17. Saltas, V., Chroneos, A., Vallianatos, F. A thermodynamic approach of self- and hetero-diffusion in GaAs: Connecting point defect parameters with bulk properties, RSC Adv., 2016, 6(58), 53324-30, Doi: 10.1039/c6ra09206c.

18. Saltas, V., Chroneos, A., Vallianatos, F. Composition and temperature dependence of self-diffusion in Si1-xGex alloys, Sci. Rep., 2017, 7(1374), Doi: 10.1038/s41598-017-01301-6.

19. Saltas, V., Chroneos, A., Vallianatos, F. Thermodynamic modelling of fast dopant diffusion in Si, J. Appl. Phys., 2018, 123, 161567-5, Doi: 10.1063/1.5001755.

20. Skordas, E.S., Sarlis, N. V., Varotsos, P.A. Applying the $\mathrm{cB} \Omega$ thermodynamical model to LiF using its equation of state obtained from high pressure diamond anvil cell measurements, Solid State Ionics, 2020, 354, 115404, Doi: 10.1016/j.ssi.2020.115404.

21. Vallianatos, F., Triantis, D., Tzanis, A., Anastasiadis, C., Stavrakas, I. Electric earthquake precursors: From laboratory results to field observations, Phys. Chem. Earth, 2004, 29, 339-51, Doi: 10.1016/j.pce.2003.12.003.

22. Vallianatos, F., Eftaxias, K. The application of the $\mathrm{cB} \Omega$ model for the calculation of the variation of the activation volume for creep with depth in the Earth's lower mantle, Phys. Earth Planet. Inter., 1992, 71, 141-6, Doi: 10.1016/0031-9201(92)90071-3.

23. Wang, F., Tange, Y., Irifune, T., and Funakoshi, K. P-V-T equation of state of stishovite up to mid-lower mantle conditions, J. Geophys. Res. 2012, 117, B06209, doi:10.1029/2011JB009100.

24. Panayiotatos, Y., Saltas, V., Chroneos, A., Vallianatos, F. Tin diffusion in germanium: a thermodynamic approach, J. Mater. Sci. Mater. Electron., 2017, 28, 9936-40, Doi: 10.1007/s10854-017-6751-7.

25. Kube, R., Bracht, H., Hüger, E., Schmidt, H., Hansen, J.L., Larsen, A.N., Ager, J.W., Haller, E.E., Geue, T., Stahn, J. Contributions of vacancies and self-interstitials to self-diffusion in silicon under thermal equilibrium and nonequilibrium conditions, Phys. Rev. B, 2013, 88, 085206, Doi: 10.1103/ PhysRevB.88.085206.

(C) 2020 by the authors. Submitted for possible open access publication under the terms and conditions of the Creative Commons Attribution (CC BY) license (http://creativecommons.org/licenses/by/4.0/). 